\title{
Knee Bony Defects Reconstruction in Revision Total Knee Arthroplasty for Aseptic Loosening
}

ISSN: 2576-8875

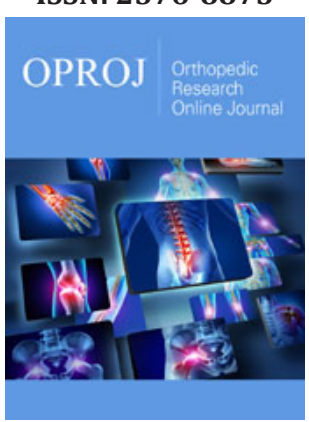

${ }^{* 1}$ Corresponding author: Ahmed A Khalifa, Orthopaedic department, Qena faculty of medicine and its university hospital, South valley university, Egypt

Submission: 佣April 02, 2019

Published: 㭗April 17, 2019

Volume 5 - Issue 4

How to cite this article: Ahmed A K ElSayed S, Hesham R, Ahmed M A. Knee Bony Defects Reconstruction in Revision Total Knee Arthroplasty for Aseptic Loosening. Ortho Res Online J. 5(3). OPROJ.000615.2019.

DOI: 10.31031/OPROJ.2019.05.000616

Copyright@: Ahmed A Khalifa, This article is distributed under the terms of the Creative Commons Attribution 4.0 International License, which permits unrestricted use and redistribution provided that the original author and source are credited.

\author{
Ahmed A Khalifa ${ }^{1 *}$, ElSayed Said ${ }^{1}$, Hesham Refae ${ }^{2}$ and Ahmed M Abdelaal ${ }^{3}$ \\ ${ }^{1}$ Orthopaedic department, Qena faculty of medicine and its university hospital, South valley \\ university, Egypt \\ ${ }^{2}$ Orthopaedic department, Aswan faculty of medicine, Aswan University, Egypt \\ ${ }^{3}$ Orthopaedic department, Assiut University hospital, Egypt
}

\begin{abstract}
Revision total knee arthroplasty (TKA) is expected to increase owing to increased number of primary knee replacement surgery done annually. Aseptic loosening considered to be one of the commonest indications for revision surgery. One of the common challenges facing the surgeon during revision surgery is how to reconstruct bony defect to obtain optimum component alignment and robust fixation. Carful pre- and intra-operative assessment is crucial and understanding of different reconstruction options side by side with sound implant selection is crucial for successful surgery. Options for bone defect reconstruction include cement with screws, morselized allograft, bulk structural allograft, modular wedges, metal augments, porous metal cones and sleeves.
\end{abstract}

Keywords: Bony defect; Reconstruction; Revision; Knee arthroplasty

\section{Introduction}

Revision total knee arthroplasty (TKA) is expected to increase dramatically owing to increasing number of primary TKA done annually [1]. Although TKA is a cost-effective procedure, revision TKA is considered more costly because of the expected higher rate of complications, prolonged surgical time, greater blood loss, prolonged hospital stays and the need for special implants [2,3]. One of the most important challenges during revision TKA, is how to deal with bone defects with its size variability and different locations, however, several techniques had been developed to compensate for these defects [4]. Soft tissue status (collateral ligaments and extensor mechanism) should be taken into consideration when evaluating the amount of bone defect and deciding how to reconstruct, a systematic approach and full orientation of the implants and reconstruction options are required for effective management of bone loss and to achieve reproducible clinical outcomes [4].

In this review we will discuss the reconstruction options for bone defects in revision TKA after failed primary TKA due to aseptic loosening.

\section{Aetiology of osteolysis and subsequent aseptic loosening}

Aseptic loosening can be the result of inadequate initial fixation, mechanical loss of fixation over time, or biologic loss of fixation caused by particle-induced osteolysis around the implant [5]. Wear debris is formed at prosthetic joint articulations, modular interfaces, and non-articulating interfaces remains the major factor limiting the survival of joint implants [6,7]. Subtle progression of tissue destruction around the implant imposes a major challenge, because signs and symptoms may not be clinically apparent until late stages of failure. The basis of periprosthetic tissue destruction, the so-called 'biologic response' to implant debris, is complex. However, this response is the outcome of multiple factors, including physical and biologic components [5,8]. At the core of the biologic response that leads to osteolysis is activation of the receptor activator of nuclear factor-B, (RANK)/RANK ligand (RANKL) axis, which is indicated by expression of RANK, RANKL, and osteoprotegerin (OPG) in periprosthetic membranes $[9,10]$. This activation ends in enhanced osteoclast recruitment and activity adjacent to bone-implant interfaces, leading to osteolysis $[11,12]$. 


\section{Patient evaluation}

History and clinical examination: A detailed history and physical examination will aid the surgeon in diagnosing the cause of TKA failure, as well as excluding other causes of failure, pain location, character, timing, and duration should be reported of pain. Competency of soft tissue envelop is very crucial to be assessed pre-operatively [4].

Laboratory investigation: These types of investigations mainly to exclude infection, serum erythrocyte sedimentation rate (normal, $20 \mathrm{mg} / \mathrm{dL}$ ) and $\mathrm{C}$ reactive protein level (normal, 10mg/ $\mathrm{dL}$ ) should be evaluated, and if elevated, knee aspiration should be done [13].

Radiological assessment: weight-bearing AP, lateral, and skyline radiographs should be done, with the radiographs of the index surgery if possible. The AP and lateral views are helpful for determine component size, fixation, and the degree of bone loss. The skyline view evaluates patellar component fixation and tracking. Full length extremity AP (hip to knee to ankle) radiographs are helpful in determining the presence of diaphyseal deformities and periarticular hardware, for assessment of the ipsilateral hip joint, and overall limb alignment [14]. MRI and CT have a high sensitivity and specificity for diagnosis of osteolysis around TKA [15]. However, these kings of imaging are not recommended as a routine owing to its increased cost and radiation exposure. 9 also, lesions can be difficult to identify due to metallic artefact effect [16].

\section{Classification of bone loss in revision TKA}

The most used classification system is The Anderson Orthopaedic Research Institute (AORI) classification [17] (Figure1) (that represents the evolution of the Engh classification [18]) which categorizes bone defects into three principle types.

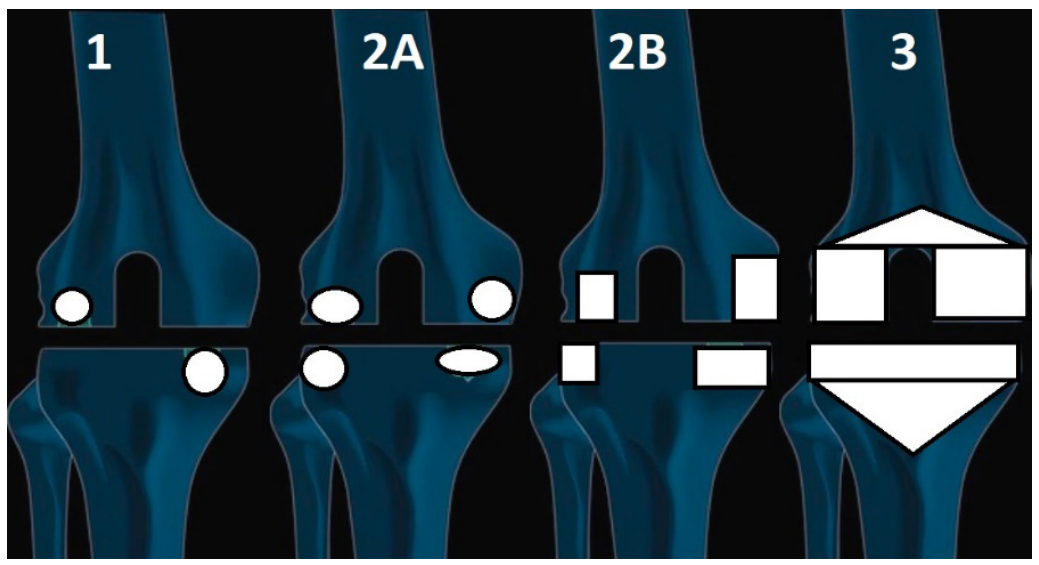

Figure 1: AORI classification for bone defect in revision TKA.

Type 1 defects when the metaphyseal bone is intact with cancellous cavitary defects without cortical defects. When considerable metaphyseal bone loss, it is considered as Type 2 defects, which can be further divided into: type $2 \mathrm{~A}$ defects affecting only one femoral condyle or tibial plateau, and type $2 \mathrm{~B}$ defects when both femoral condyles and tibial plateaus are involved. Type 3 defects if there is severe bone loss affecting major portion of the femoral condyle or tibial plateau which may violate ligaments or tendon insertions.

Re-classification of defect type usually needed intra-operative after implant and cement removal to compensate for bone lost during implant extraction. Engh argued: "the surgeon should anticipate the worst scenario, because often the defects turn out to be more severe than predicted from radiographs" [19].

\section{Reconstruction options}

Many factors should be taken into consideration when deciding if the patient can be treated non-surgically with close monitoring and follow up in combination with pharmacological options, or the osteolysis and component loosening is evident and necessitate revision, in this situation the decision should be made regarding revision of one component or the whole prothesis and how to reconstruct the bony and soft tissue defect if present.

\section{Non-surgical}

Apart from optimum fixation and improvement of the prosthetic component during primary surgery, Pharmacological strategies to prevent aseptic loosening to target the cellular components (osteoblasts and osteoclasts) that contribute to implant failure had been advocated [20].

Bisphosphonates (BPs), most commonly used in treatment of osteoporosis, had been shown to prevent early migration in TKR [21] and are associated with lower revision risk in epidemiological studies $[22,23]$.

Human clinical trials showed the efficacy of BPs in reducing particle-induced osteolysis over the first post-operative year of implanting cemented and cement less hip and knee replacement prostheses, the results were even better and more durable when treatment was started early after surgery and continued for over 6 months $[24,25]$.

A meta-analysis of 14 randomized controlled trials employing BPs after joint arthroplasty found that the protective effect of these 
drugs, may continue till the mid-term follow-up after surgery and for 18 to 70 months after drug discontinuation. BPs [26].

Giving the fact that a biological reaction to wear particles is involved in the process of osteolysis and subsequent implant loosening involving the activation of the receptor activator of nuclear factor-B, and (RANK)/RANK ligand (RANKL) axis [27], using substance whick blocks this biological reaction thought to help in prevention of early osteolysis progression in solidly fixed implants [28].

Denosumab which is another antiresorptive, a human monoclonal antibody (IgG2) which has a high binding affinity and specificity to RANKL, which may lead to preventing activation of its receptor, RANK, on the surface of osteoclast precursors and osteoclasts, with subsequent prevention of osteoclast formation and reducing its function and survival with resulting reduction in both cortical and cancellous bone resorption [29].

In a randomized, double blind placebo-controlled trial by Ledin et al. [28] where they studied 50 patients where they had TKA for osteoarthritis with an injection of either Denosumab $(60 \mathrm{mg})$ or placebo 1 day after knee replacement surgery and again after 6 months. To detect component motion, radiostereometric analysis (RSA) was performed postoperatively and after 6, 12, and 24 months after surgery. They showed that there was significantly less migration of the component in the Denosumab group compared to the control group both at 12 and 24 months. They concluded that using Denosumab after TKA will help to decrease late loosening.

\section{Surgical}

AORI type I: Impaction grafting of morselized allograft as it was originally described in revision total hip arthroplasty was modified for use in revision TKA to restore bone stock, especially in younger patients in whom future revision surgeries are expected [30]. In a study of 42 revision TKAs performed with morselized graft, Lotke et al. [31] found no evidence of failure at an average 3.8 years follow up, with an adequate graft incorporation and remodelling in all cases. In a report of 14 patients with failed impaction grafting in a revision TKA, Whiteside et al. [32] showed that biopsy specimens of the allografts had bone maturation and trabecular formation in all samples taken from areas of impacted bone graft.

Another option is to reconstruct using cement with screws is indicated If the defect is a mild contained or uncontained more than $5 \mathrm{~mm}$ but less than $10 \mathrm{~mm}$ [33]. The screws will reinforce the cement, but screws heads should be slightly below the implant [34]. The screws will help to distribute the load away from the joint line and cement bone interface. some authors [35] recommend the use of bone graft if the cement mantle below the tibial plateau is greater than $5 \mathrm{~mm}$ thick. Primary implants with the cement to fill the defects was suggested by Toms et al. [36] \& Ritter et al. [37] as an ideal method to treat these defects if it is less than $5 \mathrm{~mm}$ in breadth and depth [36,37].

AORI type II: Type 2 defect management involving the use of revision implant and the following surgical techniques.

A. Bone grafts: Impaction bone grafting as mentioned with type 1 defects can be used, when the defect is contained with bone loss more than $10 \mathrm{~mm}$ or a mild uncontained defect less than $50 \%$ of tibial plateau/condyles, this technique will help to restore bone stock [30]. It is not recommended if a cortical or uncontained defect are encountered, as the cortical rim is important for the stability of the tibial tray [38].

B. Modular metal augments (Figure 2): Metal augments are available to reconstruct either femoral or tibial defects, which comes in different shapes and sizes either cemented or cementless [39]. It is advisable to use augments in uncontained bone defects with moderate and severe bone loss $>50 \%$ and $>5 \mathrm{~mm}$ of the femoral condyle and/or tibial plateau [40]. The size of the metal augments correlates to the size of the prostheses, and should compensate for the size of the defect using different thicknesses and angles. It will be assembled to the tibial or femoral components using screws. The choice between using a wedge or block should be according to the best option closely filling the defect [41].

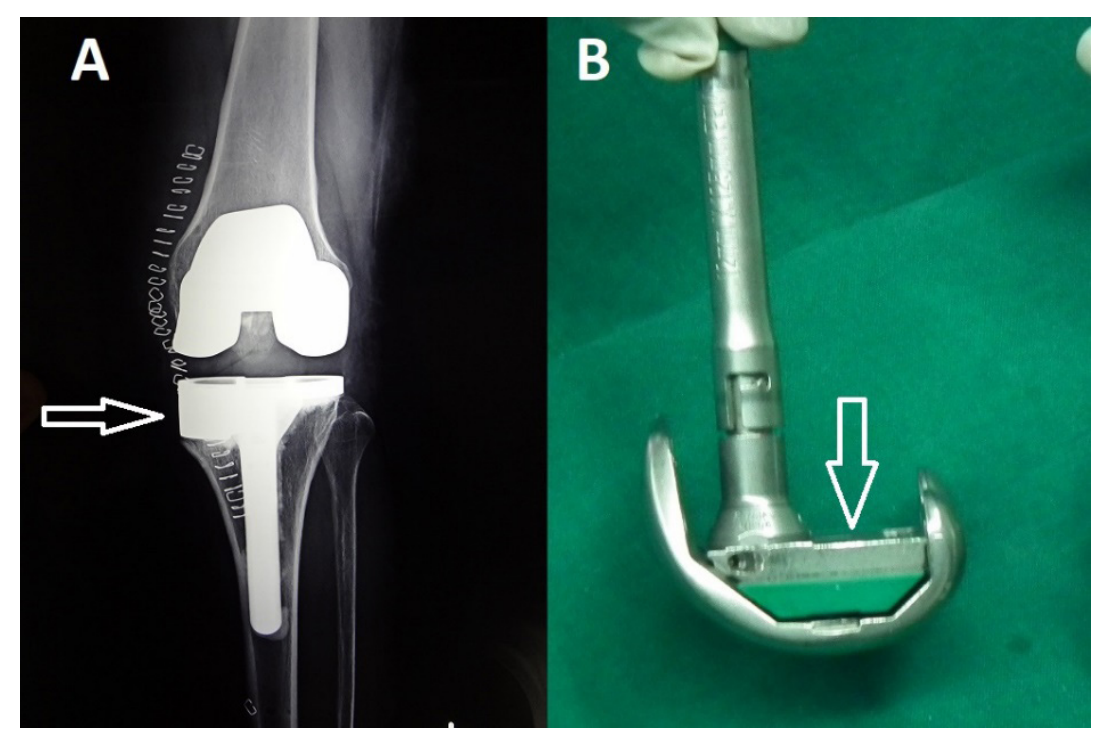

Figure 2: Metal augments, A: Medial tibial, B: Distal femoral. 
The advantages of using metal augments are the possibility to modify the implant intra-operatively with no worry of collapse, exact filling of bone defects which had been geometrically shaped with instruments, they provide stable support and transfer loading forces to the bone [42]. Modern metal augments preserve a high volumetric porosity for bone ingrowth, with low modulus of elasticity and high frictional characteristics, making this metal favourable for biologic fixation [43]. However, it carries some disadvantage, the risk of fretting and corrosion still an issue [41].

Also, the difference in elasticity between metal and bone may lead to stress shielding which may increase potential bone loss [42]. Another major problem is the limitation of the height of the augment only up to $20 \mathrm{~mm}$, which may not be suitable for larger defects [42]. Some reports showed that augmented prostheses with metal wedge showed mechanical superiority to cement alone or cement with screws in terms of resisting movement when loaded [44]. Some authors advice that better results when graft is combined with augments, with a failure of $48 \%$ when the augments were used alone compared to only $19.2 \%$ when graft and augments were used in combination [45]. The success rates in the literature using these augments range from $84 \%$ to $98 \%$ good or excellent results $[41,46]$.

AORI type III: Constrained or Megaprostheses with stems, is always the choice for such defects. Among the options to reconstruct bony defects in this type, structural allografting, cones or metaphyseal sleeves.

A. Structural allograft: It will be preferred to provide a stable and durable reconstruction of large or segmental bone deficiency in patients with a life expectancy greater than ten years, also suitable when the defects are segmental but not greater than $15 \mathrm{~mm}$ for the femur and 20-45mm for the tibiam [47].

Different types of allografts can be used, but femoral heads, distal femoral segments, and proximal tibial segments are commonly used, but it requires a vital host bone, when incorporated into the host bone, it may provide some stress protection to the implant [48]. Its usage carries some risks, as bacterial and viral disease transmission, immune response, resorption, and fatigue fracture [42]. Several reports believe showed a bulk allograft survival between $72 \%$ and $86 \%$ at eight to ten years of follow up [49]. In Eng.'s opinion the survivorship after eight years is $92 \%$ when a bulk allograft was used [50]. While, Bauman et al. [51] in their series showed limits when using this technique [51].

B. Cones (Figure 3): Filling metaphyseal defects with cones provides a base of mechanical support for the implant, with the high variability of sizes and shapes allows a good adaptability of these modules to the cavitary metaphyseal bony defects [52]. Its reported success may be attributed to the highly porous nature of the material which allows early osteointegration [53]. It also has a low modulus of elasticity of which facilitates more load transfer and preservation of bone stock [54]. It is of great hep in reconstructing large cavitary defects in which a reliable cortical shell in the face of a metaphyseal endosteal bone defect is present; when used with offset stems when necessary, may eliminate the need for bone grafting or allografting [41]. On the femoral side, using distal femoral cones help to re-establish the metaphyseal-diaphyseal junction and create a stable platform for the femoral component; which will provide structural and mechanical support [42]. After 2 years follow up, Lachiewicz et al. [55] found that tantalum cones were fully integrated. In the Mayo Clinic experience, tantalum cones were found to have osteintegrated in $100 \%$ of tibial [55] and femoral cases [53].

Figure 3: Trabecular metal cone.

C. Metaphyseal sleeves (Figure 4): Femoral sleeves are indicated in patients where there is a metaphyseal deficiency, if there is a limited bony support for the femoral component and if there is difficulty controlling the rotation of the femoral implant, femoral sleeves may also be beneficial when there is significant posterior femoral condyle bone loss, as they can add rotational stability of the implant [42]. Unlike tantalum cones, where the implant is fixed to the cone by cement, metaphyseal sleeves are fixed to the implant with a morse taper [4]. The most common complication from the use of metaphyseal sleeves is fracture when broaching the sleeves in the tibia or the femur [56]. A prospective study examined outcomes of 83 revision TKAs with metaphyseal femoral and tibial sleeves at a minimum 2-year follow up [57]. None of the knees demonstrated progressive radiolucent lines around the 
metaphyseal sleeves. At final follow-up, two tibial sleeves $(2.7 \%)$ required revision for aseptic loosening.

\section{Conclusion}

Bony defect reconstruction during revision TKA is challenging. Early detection of osteolysis with the possibility of pharmacological treatment before frank implant loosening may have a role. A detailed patient history, physical examination, and radiographic assessment are essential for surgical planning. Care should be taken when removing the implants to avoid further iatrogenic bone loss. Different options and technique are present to reconstruct tibial and femoral bony defect encountered during revision surgery. Careful selection between these options and judicious implant use will help to achieve a reproducible radiological and clinical outcomes.

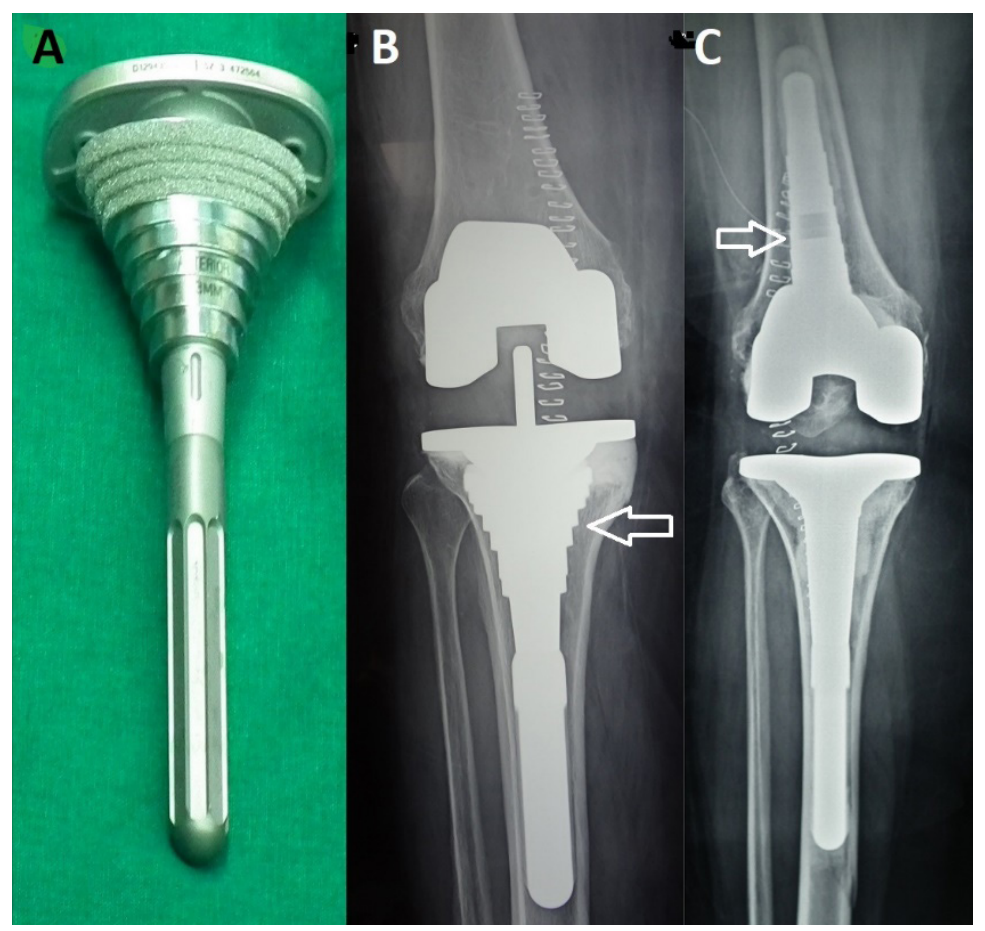

Figure 4: Sleeves, A\&B: Tibial, C: Femoral.

\section{References}

1. Bozic KJ, Kurtz SM, Lau E, Ong K, Chiu V, et al. (2010) The epidemiology of revision total knee arthroplasty in the United States. Clin Orthop Relat Res 468(1): 45-51.

2. Kallala R, Vanhegan I, Ibrahim M, Sarmah S, Haddad F (2015) Financial analysis of revision knee surgery based on NHS tariffs and hospital costs: does it pay to provide a revision service? The bone \& Joint Journal 97(2): 197-201.

3. Kasmire KE, Rasouli MR, Mortazavi SJ, Sharkey PF, Parvizi J (2014) Predictors of functional outcome after revision total knee arthroplasty following aseptic failure. The Knee 21(1): 264-267.

4. Sheth NP, Bonadio MB, Demange MK (2017) Bone loss in revision total knee arthroplasty: evaluation and management. JAAOS-Journal of the American Academy of Orthopaedic Surgeons 25(5): 348-357.

5. Abu-Amer Y, Darwech I, Clohisy JC (2007) Aseptic loosening of total joint replacements: mechanisms underlying osteolysis and potential therapies. Arthritis Research \& Therapy 9(1): S6.

6. Marshall A, Ries MD, Paprosky W (2008) How prevalent are implant wear and osteolysis, and how has the scope of osteolysis changed since 2000? JAAOS-Journal of the American Academy of Orthopaedic Surgeons 16: S1-S6.

7. Goldfing S, Clark C, Wright $T$ (1993) The problem in tota1 joint arthroplasty: Aseptic loosening [Editorial]. J Bone Joint Surg A 75(6): 799-801.
8. Maloney WJ, Smith RL (1995) Periprosthetic osteolysis in total hip arthroplasty: the role of particulate wear debris. JBJS 77 (9): 1448-1461.

9. Crotti TN, Smith MD, Findlay D, Zreiqat H, Ahern MJ, et al. (2004) Factors regulating osteoclast formation in human tissues adjacent to periimplant bone loss: Expression of receptor activator NFKB, RANK ligand and osteoprotegerin. Biomaterials 25(4): 565-573.

10. Gehrke T, Sers C, Morawietz L, Fernahl G, Neidel J, et al. (2003) Receptor activator of nuclear factor $\mathrm{\kappa B}$ ligand is expressed in resident and inflammatory cells in aseptic and septic prosthesis loosening. Scandinavian Journal of Rheumatology 32(5): 287-294.

11. Khosla S (2001) Minireview: The opg/rankl/rank system. Endocrinology 142(12): 5050-5055.

12. Abu-Amer Y (2005) Advances in osteoclast differentiation and function. Current Drug Targets-Immune, Endocrine \& Metabolic Disorders 5(3): 347-355.

13. Della Valle CJ, Sporer SM, Jacobs JJ, Berger RA, Rosenberg AG, etal. (2007) Preoperative testing for sepsis before revision total knee arthroplasty. The Journal of Arthroplasty 22(6): 90-93.

14. Nadaud MC, Fehring TK, Fehring K (2004) Underestimation of osteolysis in posterior stabilized total knee arthroplasty. The Journal of Arthroplasty 19(1): 110-115.

15. Minoda Y, Yoshida T, Sugimoto K, Baba S, Ikebuchi M, et al. (2014) Detection of small periprosthetic bone defects after total knee arthroplasty. The Journal of Arthroplasty 29(12): 2280-2284. 
16. Vessely MB, Frick MA, Oakes D, Wenger DE, Berry DJ (2006) Magnetic resonance imaging with metal suppression for evaluation of periprosthetic osteolysis after total knee arthroplasty. The Journal of Arthroplasty 21(6): 826-831.

17. Engh GA, Ammeen DJ (1998) Classification and preoperative radiographic evaluation: knee. Orthopedic Clinics of North America 29(2): 205-217.

18. Engh G, Ammeen D (1999) Bone loss with revision total knee arthroplasty: defect classification and alternatives for reconstruction. Instructional Course Lectures 48: 167-175.

19. Engh GA, Herzwurm PJ, Parks NL (1997) Treatment of major defects of bone with bulk allografts and stemmed components during total knee arthroplasty. JBJS 79(7): 1030-1039.

20. Cavalli L, Brandi ML (2013) Periprosthetic bone loss: diagnostic and therapeutic approaches. F1000 Research 2: 266.

21. Hilding M, Aspenberg P (2006) Postoperative clodronate decreases prosthetic migration: 4-year follow-up of a randomized radiostereometric study of 50 total knee patients. Acta Orthopaedical 77(6): 912-916.

22. Teng S, Yi C, Krettek C, Jagodzinski M (2015) Bisphosphonate use and risk of implant revision after total hip/knee arthroplasty: a metaanalysis of observational studies. PloS One 10(10): e0139927.

23. Namba RS, Inacio MC, Cheetham TC, Dell RM, Paxton EW, et al (2016) Lower total knee arthroplasty revision risk associated with bisphosphonate use, even in patients with normal bone density. The Journal of Arthroplasty 31(2): 537-541.

24. Bhandari M, Bajammal S, Guyatt GH, Griffith L, Busse JW, et al. (2005) Effect of bisphosphonates on periprosthetic bone mineral density after total joint arthroplasty: a meta-analysis. JBJS 87(2): 293-301.

25. Wilkinson JM, Eagleton AC, Stockley I, Peel NF, Hamer AJ, et al. (2005) Effect of pamidronate on bone turnover and implant migration after total hip arthroplasty: a randomized trial. Journal of Orthopaedic Research 23(1): 1-8.

26. Lin T, Yan SG, Cai XZ, Ying ZM (2012) Bisphosphonates for periprosthetic bone loss after joint arthroplasty: a meta-analysis of 14 randomized controlled trials. Osteoporosis International 23(6): 1823-1834.

27. Horiki M, Nakase T, Myoui A, Sugano N, Nishii T, et al. (2004) Localization of RANKL in osteolytic tissue around a loosened joint prosthesis. Journal of Bone and Mineral Metabolism 22(4): 346-351.

28. Ledin H, Good L, Aspenberg P (2017) Denosumab reduces early migration in total knee replacement. Acta Orthopaedica 88(3): 255-258.

29. Kostenuik PJ (2005) Osteoprotegerin and RANKL regulate bone resorption, density, geometry and strength. Current Opinion in Pharmacology 5(6): 618-625.

30. Suárez-Suárez M, Murcia A, Maestro A (2002) Filling of segmental bone defects in revision knee arthroplasty using morsellized bone grafts contained within a metal mesh. Acta Orthopaedica Belgica 68(2): 163167.

31. Lotke PA, Carolan GF, Puri N (2006) Impaction grafting for bone defects in revision total knee arthroplasty. Clinical Orthopaedics and Related Research 446: 99-103.

32. Whiteside LA, Bicalho PS (1998) Radiologic and histologic analysis of morselized allograft in revision total knee replacement. Clinical Orthopaedics and Related Research (357): 149-156.

33. Mauffrey C, Barlow BT, Smith W (2015) Management of segmental bone defects. JAAOS-Journal of the American Academy of Orthopaedic Surgeons 23(3): 143-153.

34. Scott RD (1995) Bone loss: prosthetic and augmentation method Orthopedics 18(9): 923-926.

35. Dorr L (1989) Bone grafts for bone loss with total knee replacement. The Orthopedic Clinics of North America 20(2): 179-187.
36. Toms A, Barker R, McClelland D, Chua L, Spencer-Jones R, et al. (2009) Repair of defects and containment in revision total knee replacement: a comparative biomechanical analysis. The Journal of Bone and Joint Surgery British 91(2): 271-277.

37. Ritter MA, Keating EM, Faris PM (1993) Screw and cement fixation of large defects in total knee arthroplasty: a sequel. The Journal of Arthroplasty 8(1): 63-65.

38. Toms AD, Barker RL, Jones RS, Kuiper JH (2004) Impaction bone-grafting in revision joint replacement surgery. JBJS 86(9): 2050-2060.

39. Radnay CS, Scuderi GR (2006) Management of bone loss: augments, cones, offset stems. Clinical Orthopaedics and Related Research 446: 83-92.

40. Whittaker J, Dharmarajan R, Toms A (2008) The management of bone loss in revision total knee replacement. The Journal of Bone and Joint Surgery British 90(8): 981-987.

41. Patel J, Masonis J, Guerin J, Bourne R, Rorabeck C (2004) The fate of augments to treat type- 2 bone defects in revision knee arthroplasty. The Journal of Bone and Joint Surgery British 86(2): 195-199.

42. Panegrossi G, Ceretti M, Papalia M, Casella F, Favetti F, et al. (2014) Bone loss management in total knee revision surgery. International Orthopaedics 38(2): 419-427.

43. Tigani D, Sabbioni G, Raimondi A (2009) Early aseptic loosening of a porous tantalum knee prosthesis. Chir Organi Mov 93(3): 187-191.

44. Brooks PJ, Walker PS, Scott RD (1984) Tibial component fixation in deficient tibial bone stock. Clinical Orthopaedics and Related Research (184): 302-308.

45. Hockman DE, Ammeen D, Engh GA (2005) Augments and allografts in revision total knee arthroplasty: usage and outcome using one modular revision prosthesis. The Journal of arthroplasty 20(1): 35-41.

46. Lucey SD, Scuderi GR, Kelly MA, Insall JN (2000) A practical approach to dealing with bone loss in revision total knee arthroplasty. Orthopedics 23(10): 1036-1041.

47. McAllister DR, Joyce MJ, Mann BJ, Vangsness CT (2007) Allograft update: the current status of tissue regulation, procurement, processing, and sterilization. The American Journal of Sports Medicine 35(12): 21482158.

48. Dennis DA, Little LR (2005) The structural allograft composite in revision total knee arthroplasty. Orthopedics 28(9): 1005-1007.

49. Dennis DA (2002) The structural allograft composite in revision total knee arthroplasty. The Journal of Arthroplasty 17(4): 90-93.

50. Engh GA, Ammeen DJ (2007) Use of structural allograft in revision total knee arthroplasty in knees with severe tibial bone loss. JBJS 89(12): 2640-2647.

51. Bauman RD, Lewallen DG, Hanssen AD (2009) Limitations of structural allograft in revision total knee arthroplasty. Clinical Orthopaedics and Related Research 467(3): 818-824.

52. Kamath AF, Lewallen DG, Hanssen AD (2015) Porous tantalum metaphyseal cones for severe tibial bone loss in revision knee arthroplasty: a five to nine-year follow-up. JBJS 97(3): 216-223.

53. Howard JL, Kudera J, Lewallen DG, Hanssen AD (2011) Early results of the use of tantalum femoral cones for revision total knee arthroplasty. JBJS 93(5): 478-484.

54. Maccauro G, Iommetti PR, Muratori F, Raffaelli L, Manicone PF, et al. (2009) An overview about biomedical applications of micron and nano size tantalum. Recent Patents on Biotechnology 3(3): 157-165.

55. Lachiewicz PF, Bolognesi MP, Henderson RA, Soileau ES, Vail TP (2012) Can tantalum cones provide fixation in complex revision knee arthroplasty? Clinical Orthopaedics and Related Research 470(1): 199204. 
56. Alexander GE, Bernasek TL, Crank RL, Haidukewych GJ (2013) Cementless metaphyseal sleeves used for large tibial defects in revision total knee arthroplasty. The Journal of arthroplasty 28(4): 604-607.
57. Huang R, Barrazueta G, Ong A, Orozco F, Jafari M, et al. (2014) Revision total knee arthroplasty using metaphyseal sleeves at short-term followup. Orthopedics 37(9): e804-e809.

For possible submissions Click below: 